\title{
Was man über Nahtanker zur Rekonstruktion der Rotatorenmanschette wissen sollte: wann welche Ankerkombination?
}

\author{
Atesch Ateschrang, Stephan Riedmann, Steffen Schröter, Ulrich Stöckle
}

\section{Zusammenfassung}

Durch die Anwendung erster metallischer Ankersysteme wurde eine erhebliche Vereinfachung sowohl offener als auch arthroskopischer Operationen möglich. Mittlerweile wurden die Werkstoffe und das Design moderner Ankersysteme weiterentwickelt, sodass sich ein breiter Anwendungsbereich an der oberen und unteren Extremität etabliert hat. Prinzipiell können Fadenanker und knotenlose Anker differenziert werden. In der operativen Therapie der Rotatorenmanschettenpathologie können sowohl Fadenanker als auch knotenlose Anker separat und in Kombination angewandt werden. In diesem Artikel werden die wesentlichen Differenzierungsmerkmale der unterschiedlichen Anker dargestellt und zahlreiche Ankersysteme bez. ihrer Materialbeschaffenheit und technischen Merkmale inkl. der biomechanischen Ausreißkräfte systematisch zusammengefasst.

\section{What You Need to Know About Suture Anchors in Rotator Cuff Reconstruction: When to Use Which Combination?}

The use of the first metallic anchor system initiated an appreciable simplification for both open and arthroscopic operations. In the meantime materials and designs have been further developed and thus a broad range of applications in not only the upper but also the lower limbs has become established. In principle, one can distinguish between suture anchors and knotless anchors. In the surgical treatment of rotator cuff pathologies both suture anchors and knotless anchors can be used separately and in combination. In the present article we present a systematic summary of the major special distinguishing features of the various types of anchors and numerous other anchor systems with regard to their material properties and technical features, including their biomechanical pull-out forces.

\section{Abkürzungen}

RM Rotatorenmanschette

SLAP Superior Labrum Anterior to Posterior

\section{Einleitung}

Die arthroskopische und offene RM-Rekonstruktion wurde durch die Entwicklung von Nahtankern erheblich erleichtert. Die ersten metallischen Ankersysteme mit widerhakenähnlichem Design haben es erstmals möglich gemacht, auch bei osteoporotischer Knochenqua-

OP-JOURNAL 2013; 29: 261-266

(C) Georg Thieme Verlag KG Stuttgart · New York DOI http://dx.doi.org/10.1055/s-0034-1368172 lität des Humeruskopfs eine sichere Verankerung zu gewährleisten. Allerdings kann man nicht automatisch davon ausgehen, dass alle Ankersysteme gleichermaßen für unkomplizierte RM-Läsionen geeignet sind, wie etwa für stark retrahierte bzw. komplexe und schwer erreichbare Sehnenläsionen, wie der Subscapularissehne.

Die Anforderungen an eine RM-Rekonstruktion sind ganz anders zu bewerten wie bspw. die einer glenohumeralen Stabilisierung im Rahmen einer KapselLabrum-Rekonstruktion. Die RM-Nahtankersysteme sollen insbesondere bei osteoporotischer Knochenqualität durch die Nähte die Sehne ausreichend lange an den Humeruskopf fixieren bis zur sicheren osteotendinösen Einheilung [1].
Die biomechanischen Anforderungen werden durch teilweise schlechter Sehnenqualität wie reduziertem Sehnendurchmesser und/oder Brüchigkeit sowie Muskelatrophie und dadurch erhöhte Primärspannung der RM-Rekonstruktion erschwert [2]. Auch die postoperative Nachbehandlung in Bezug auf Ruhigstellungsdauer, Position des ruhig gestellten Schultergelenks und der erlaubten KG-Intensität spielen eine relevante Rolle mit daraus resultierender biomechanischer Beanspruchung der Nahtanker [2].

Durch die Entwicklung der arthroskopischen Rekonstruktionsmöglichkeiten wurden die Nahtanker in Sachen Material, Formgebung, Ösenform und Ösenbeschaffenheit sowie Nahtmaterial weiterentwickelt mit einer heute für den Chirurgen großen Aus-(qual-)wahlmöglichkeit $[1,3,4]$.

Ziel dieses Artikels ist es, einen Überblick über die verfügbaren Nahtankersysteme zu geben. Ein Anspruch auf Vollständigkeit sämtlicher verfügbarer Anker besteht allerdings nicht, um diesen Artikel übersichtlich $\mathrm{zu}$ gestalten. Aufbauend auf diesen Erkenntnissen und klinischen Erfahrungen werden für konkrete klinische Problemkonstellationen Vorschläge für das praktische Vorgehen abgeleitet, welche Ankerkonstellationen sinnvoll eingesetzt werden können.

\section{Hauptteil}

Historisch wurde die RM-Rekonstruktion vor der Verfügbarkeit von Nahtankern durch transossäre Nähte mittels Bohrlöcher durch das Tuberculum-majus-Massiv realisiert [2,5].

Dabei wurden zunächst knöcherne Fadentunnel mittels Bohrer, Knochenahlen, Stanzen oder Pfriem angelegt, um anschließend die Nähte durch das Tuberculum-majus-Massiv zu shutteln. Diesbez. wurde die Problematik des knöcher- 


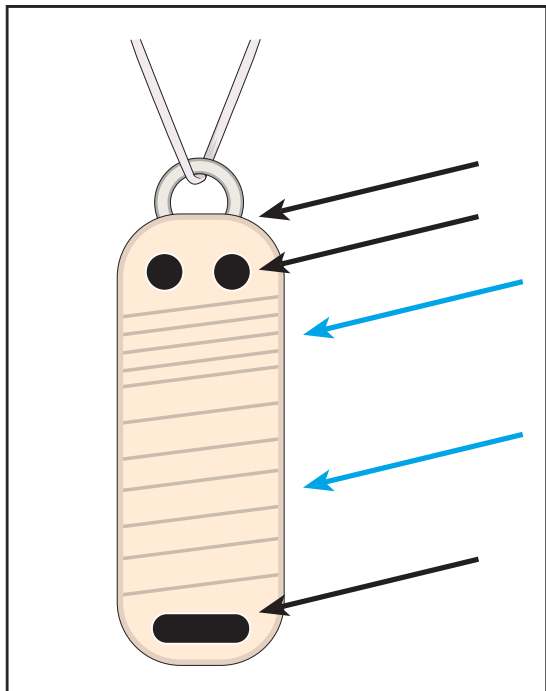

Abb. 1 Schema eines Fadenankers. Schwarze Pfeile markieren die möglichen Orte für die Platzierung der Öse. Blaue Pfeile markieren das Gewinde, welches durchgehend ausgebildet sein kann oder dual aufgebaut ist mit einem kortikalen (geringe Gewindesteigung) und spongiösen (große Gewindesteigung) Abschnitt. Hier ist schemenhaft ein zylindrisch imponierender Anker mit einer stumpfen Spitze abgebildet, wobei unterschiedlichste Formgebungen möglich sind.

nen Einschneidens der Nähte diskutiert, sodass einige Autoren ein distaleres Ausleiten der Fäden empfahlen. Biomechanische Vergleichsuntersuchungen zeigten, dass die RM-Rekonstruktion mit Nahtankern der transossären Technik unabhängig von der Knochenqualität überlegen war. Zusätzlich haben Nahtanker deutliche Vorteile, da sie sowohl für die offene als auch für das arthroskopische Vorgehensweise gleichermaßen geeignet sind $[5,6]$.

Die ersten verfügbaren Anker wurden für die glenohumerale Stabilisierung entwickelt. Grundsätzlich kann man den im Knochen befindlichen Anker, das am Anker befestigte Faden- bzw. Nahtmaterial und die für den jeweiligen Anker spezifischen Implantationsinstrumente differenzieren. Aufgrund der kortikalen Knochenqualität des Glenoids und der Größe müssen glenoidal zu implantierende Anker klein sein und im kortikalen Knochen festen Halt finden. Die unterschiedliche Charakteristik des Tuberculum majus und des Glenoids bedingen deutliche Größen- und Designunterschiede. Beispielsweise ist das Durchschnittsalter der Patienten mit RM-Rekonstruktion deutlich höher als das der Patienten mit Labrum- oder SLAP-Repair. Man wird dadurch häufiger mit osteoporotischer Knochenqualität

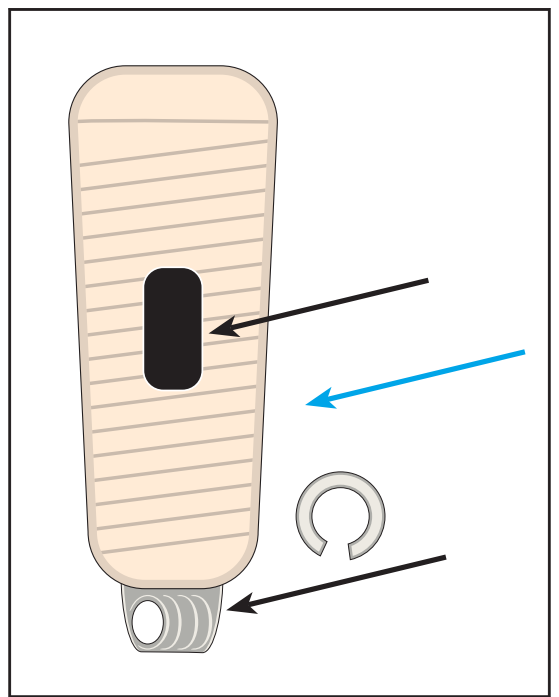

Abb. 2 Schema eines knotenfreien Ankers. Schwarze Pfeile markieren die möglichen Orte für die Platzierung der Öse zur Aufnahme der zu fixierenden Fäden. Blaue Pfeile markieren das Gewinde, welches durchgehend ausgebildet sein kann (einzuschraubende Anker). Einzuschlagende Anker weisen hingegen ein orthogonal verlaufendes Profil zur Ankerlängsachse auf.

oder gar zystischen Veränderungen im Rahmen von RM-Rekonstruktionen konfrontiert.

Nahtanker unterscheiden sich durch Material, Formgebung, Gewindedesign, Ösenform- und Positionierung, Ösenbeschaffenheit sowie Fadenmaterial.

Muss man sich als Chirurg für einen Anker entscheiden, so hat man eine große Auswahlmöglichkeit. Grundsätzlich kann man die nachfolgenden Ankermerkmale unterscheiden (Abb. 1 und 2):

1. Länge und Durchmesser

2. Implantationstechnik und dadurch unterschiedliche Implantationsinstrumente (mehr oder weniger aufwendig):

a) Schraub- bzw. „screw-in“-Anker. Ohne oder mit Öse an der Ankerspitze (insbesondere für die 2. Nahtreihe)

b) Einzuschlagende bzw. „tap-in“-Anker

- einfache Stiftanker, die durch ihr Außenprofil im Knochen ausreichend Haltekraft bieten. Ohne oder mit Öse an der Ankerspitze (insbesondere für die 2. Nahtreihe)

- widerhakenähnliche Spreizanker

- fadenbasierender Knochenanker
Zusammenfassend einfache oder schwierige Implantationstechnik (Lernkurve) mit mehr oder weniger ausgeprägter Fehleranfälligkeit sowie arthroskopische Anwendbarkeit.

3. Ösenform und Anzahl sowie Lokalisation (zentral, dezentral, Ankerspitze oder Ende, etc.)

4. Anzahl der Fäden sowie Materialbeschaffenheit (resorbierbar/nicht resorbierbar/partiell resorbierbar)

5. Ankermaterial (resorbierbar/nicht resorbierbar/partiell resorbierbar)

6. Strahlendurchlässigkeit (ja/nein/partiell)

\section{Revisionsmöglichkeit}

Abhängig vom oben erwähnten Ankerdesign und den Ankerdimensionen ist der Betrachter geneigt, die knöchernen Auszugskräfte des jeweiligen Ankersystems subjektiv abzuschätzen. Biomechanische Untersuchungen zur Bestimmung der Ankerausreißkraft wurden von Barber et al. durchgeführt $[1,3,4]$. Tab. 1 bietet einen Überblick über industriell verfügbare Ankersysteme. Einige dieser Anker wurden biomechanisch getestet. Die biomechanischen Untersuchungen beruhen auf standardisierten Implantationsbedingungen. Alle Anker wurden mit demselben Fadenmaterial auf Ausreißfestigkeit untersucht: UHMWPE (Polyethylen mit ultrahohem Molekulargewicht). Die Anker wurden in den metaphysären Knochenabschnitt implantiert. Dabei wurde für jeden Anker (je 10 Stück) auch der kortikale Knochen entfernt, sodass für jeden Anker die spongiöse und kortikale Knochenausreißfestigkeit bestimmt wurde. Diese Differenzierung ist insofern wichtig, um in Abhängigkeit von der lokalen Knochenbeschaffenheit entscheiden zu können, welcher Anker im Knochen die beste Ausreißfestigkeit bietet. Dieses Messprotokoll soll das Implantatversagen zwischen Knochen und Anker (Knochen-Anker-Interface) sowie zwischen Anker und Fadenmaterial (Anker-Faden-Interface bzw. Öse) testen, im Sinne einer Summationsmessung.

Bei der aktuellsten Untersuchungsreihe wurden zyklische Untersuchungen durchgeführt [1]. Jeder Anker wurde in mind. 5 unterschiedlichen Femora getestet. Die Testreihen wurden alle bei Raumtemperatur durchgeführt. Ein speziell präparierter Aluminiumrahmen wurde zur Fixierung des Femurs verwendet. Der Auszugsvektor war parallel und direkt über dem Anker. Das Testprotokoll umfasste eine Vorspannung von $10 \mathrm{~N}$ (entspricht einer Zugkraft von 
Tab. 1 Industriell verfügbare Ankersysteme.

\begin{tabular}{|c|c|c|c|c|c|c|c|}
\hline Anker & Material & Faden & Armierung & $\begin{array}{l}\text { Kern- } \\
\text { durchmesser }\end{array}$ & $\begin{array}{l}\text { Außen- } \\
\text { durchmesser }\end{array}$ & Länge & Zusatzinformation \\
\hline ReelX & PEEK & $\begin{array}{l}\text { Nr. } 2 \text { Force } \\
\text { Fiber }\end{array}$ & doppelt & konisch & $5,5 \mathrm{~mm}$ & $19,4 \mathrm{~mm}$ & stufenweise spannen \\
\hline $\begin{array}{l}\text { Footprint ul- } \\
\text { tra }(4,5 \mathrm{~mm} / \\
5,5 \mathrm{~mm})\end{array}$ & PEEK & $\begin{array}{l}\text { Aufnahme } \\
\text { von } 4 \mathrm{Nr} \text {.- } \\
\text { 2-Fäden }\end{array}$ & knotenfrei & $3,0 \mathrm{~mm} / 3,9 \mathrm{~mm}$ & $\begin{array}{l}4,5 \mathrm{~mm} / \\
5,5 \mathrm{~mm}\end{array}$ & $21 \mathrm{~mm}$ & $\begin{array}{l}\text { laterale Nahtreihe, } \\
\text { einzuschlagen }\end{array}$ \\
\hline $\begin{array}{l}\text { TwinFix } \\
(4,5 \mathrm{~mm} / \\
5,5 \mathrm{~mm})\end{array}$ & $\begin{array}{l}\text { PEEK } \\
\text { Titan } \\
\text { PLLA/HA }\end{array}$ & Nr.-2-Fäden & $\begin{array}{l}\text { 4,5 mm: } \\
\text { doppelt } \\
\text { 5,5 mm: } \\
\text { doppelt oder } \\
\text { 3-fach }\end{array}$ & $\begin{array}{l}\text { PEEK und PLLA/ } \\
\text { HA: } 2,8 \mathrm{~mm} / \\
3,2 \mathrm{~mm} \\
\text { Titan: } 3,2 \mathrm{~mm} / \\
3,5 \mathrm{~mm}\end{array}$ & $\begin{array}{l}4,5 \mathrm{~mm} / \\
5,5 \mathrm{~mm}\end{array}$ & $19 \mathrm{~mm}$ & $\begin{array}{l}\text { mediale Nahtreihe, } \\
\text { einzuschrauben }\end{array}$ \\
\hline $\begin{array}{l}\text { TwinFix Ultra } \\
(6,5 \mathrm{~mm})\end{array}$ & $\begin{array}{l}\text { PEEK } \\
\text { Titan } \\
\text { PLLA/HA }\end{array}$ & Nr.-2-Fäden & $\begin{array}{l}\text { doppelt oder } \\
\text { 3-fach }\end{array}$ & $\begin{array}{l}\text { PEEK und PLLA/ } \\
\text { HA: } 3,5 \mathrm{~mm} \\
\text { Titan: } 4,3 \mathrm{~mm}\end{array}$ & $6,5 \mathrm{~mm}$ & $19 \mathrm{~mm}$ & $\begin{array}{l}\text { mediale Nahtreihe, } \\
\text { einzuschrauben, } \\
\text { durchgehendes } \\
\text { Gewinde }\end{array}$ \\
\hline $\begin{array}{l}\text { Morphix } \\
(4,5 \mathrm{~mm} / \\
5,5 \mathrm{~mm})\end{array}$ & PEEK & Nr.-2-Fäden & doppelt & $\begin{array}{l}3,75 \mathrm{~mm} / \\
4,75 \mathrm{~mm}\end{array}$ & $\begin{array}{l}\text { gespreizt } \\
15 \mathrm{~mm}\end{array}$ & $\begin{array}{l}\text { gespreizt } \\
11,25 \mathrm{~mm}\end{array}$ & $\begin{array}{l}\text { Spreizanker, mediale } \\
\text { Nahtreihe }\end{array}$ \\
\hline CrossfT BC & $\begin{array}{l}77 \% \text { PLDLA } \\
23 \% \text { micro- } \\
\text { TCP }\end{array}$ & Nr.-2-Fäden & $\begin{array}{l}\text { doppelt oder } \\
\text { 3-fach }\end{array}$ & $3,8 \mathrm{~mm}$ & $5,5 \mathrm{~mm}$ & $17 \mathrm{~mm}$ & $\begin{array}{l}\text { mediale Nahtreihe, } \\
\text { kortikales und spon- } \\
\text { giöses Gewinde }\end{array}$ \\
\hline JuggerKnot & $\begin{array}{l}\text { geflochtener } \\
\text { Polyester }\end{array}$ & Nr.-2-Fäden & doppelt & $2,9 \mathrm{~mm}$ & $2,9 \mathrm{~mm}$ & $\begin{array}{l}25 \text { mm } \\
\text { Faden- } \\
\text { schlauch }\end{array}$ & $\begin{array}{l}\text { mediale Reihe wei- } \\
\text { cher, flexibler Anker } \\
\text { zu } 100 \% \text { aus Faden- } \\
\text { material }\end{array}$ \\
\hline $\begin{array}{l}\text { Healicoil } \\
(4,5 \mathrm{~mm} / \\
5,5 \mathrm{~mm})\end{array}$ & PEEK & Nr.-2-Fäden & $\begin{array}{l}\text { 4,5 mm: } \\
\text { doppelt } \\
\text { 5,5 mm: } \\
\text { doppelt oder } \\
\text { 3-fach }\end{array}$ & $\begin{array}{l}4,5 \mathrm{~mm}: 3 \mathrm{~mm} \\
5,5 \mathrm{~mm}: 4 \mathrm{~mm}\end{array}$ & $\begin{array}{l}4,5 \mathrm{~mm} / \\
5,5 \mathrm{~mm}\end{array}$ & $19 \mathrm{~mm}$ & $\begin{array}{l}\text { mediale Nahtreihe, } \\
\text { offene Ankerarchi- } \\
\text { tektur }\end{array}$ \\
\hline $\begin{array}{l}\text { Quattro X } \\
(5,5 \mathrm{~mm} / \\
6,5 \mathrm{~mm})\end{array}$ & PEEK & Nr.-2-Fäden & doppelt & $3,75 \mathrm{~mm} / 4 \mathrm{~mm}$ & $\begin{array}{l}5,5 \mathrm{~mm} / \\
6,5 \mathrm{~mm}\end{array}$ & $16 \mathrm{~mm}$ & $\begin{array}{l}\text { mediale Nahtreihe, } \\
\text { stumpfe Ankerspitze, } \\
\text { konische Form, } \\
\text { Fadenöse bricht bei } \\
\text { Ankerbruch }\end{array}$ \\
\hline $\begin{array}{l}\text { Quattro Link } \\
\text { (4,5 mm/ } \\
5,5 \mathrm{~mm})\end{array}$ & PEEK & $\begin{array}{l}\text { Aufnahme } \\
\text { von } 4 \text { Nr.-2- } \\
\text { Fäden }\end{array}$ & knotenfrei & $3,6 \mathrm{~mm} / 4,5 \mathrm{~mm}$ & $\begin{array}{l}4,5 \mathrm{~mm} / \\
5,5 \mathrm{~mm}\end{array}$ & $23 \mathrm{~mm}$ & $\begin{array}{l}\text { laterale Nahtreihe, } \\
\text { knotenfreier Anker }\end{array}$ \\
\hline $\begin{array}{l}\text { Healix } \\
(5,5 \mathrm{~mm} / \\
6,5 \mathrm{~mm})\end{array}$ & $\begin{array}{l}\text { PEEK } \\
\text { BR } \\
\text { Titan }\end{array}$ & Nr.-2-Fäden & doppelt & $3,9 \mathrm{~mm} / 4,5 \mathrm{~mm}$ & $\begin{array}{l}5,5 \mathrm{~mm} / \\
6,5 \mathrm{~mm}\end{array}$ & $18 \mathrm{~mm}$ & $\begin{array}{l}\text { mediale Nahtreihe, } \\
\text { kortikales und spon- } \\
\text { giöses Gewinde }\end{array}$ \\
\hline $\begin{array}{l}\text { Corkscrew } \\
\text { FT }(4,5 \mathrm{~mm} / \\
5,5 \mathrm{~mm} \text { und } \\
6,5 \mathrm{~mm})\end{array}$ & $\begin{array}{l}\text { PEEK } \\
\text { PLLA } \\
\text { Titan } \\
\text { Biocomposite }\end{array}$ & Nr.-2-Fäden & doppelt & $\begin{array}{l}3,5 \mathrm{~mm} / 4,5 \mathrm{~mm} \\
\text { und } 5,5 \mathrm{~mm}\end{array}$ & $\begin{array}{l}4,5 \mathrm{~mm} / \\
5,5 \mathrm{~mm} \text { und } \\
6,5 \mathrm{~mm}\end{array}$ & $\begin{array}{l}15 \mathrm{~mm} / \\
16 \mathrm{~mm}\end{array}$ & mediale Nahtreihe \\
\hline $\begin{array}{l}\text { PushLock } \\
(3,5 \mathrm{~mm} / \\
4,5 \mathrm{~mm})\end{array}$ & $\begin{array}{l}\text { PEEK } \\
\text { PLLA } \\
\text { Biocomposite }\end{array}$ & $\begin{array}{l}\text { Aufnahme } \\
\text { von } 4 \text { Nr.-2- } \\
\text { Fäden }\end{array}$ & knotenfrei & $2,9 \mathrm{~mm} / 4,5 \mathrm{~mm}$ & $\begin{array}{l}3,5 \mathrm{~mm} / \\
4,5 \mathrm{~mm}\end{array}$ & $\begin{array}{l}14 \mathrm{~mm} / \\
18,5 \mathrm{~mm}\end{array}$ & $\begin{array}{l}\text { laterale Nahtreihe, } \\
\text { einzuschlagen }\end{array}$ \\
\hline $\begin{array}{l}\text { SwiveLock C } \\
(4,75 \mathrm{~mm} / \\
5,5 \mathrm{~mm})\end{array}$ & $\begin{array}{l}\text { PEEK } \\
\text { PLLA } \\
\text { Biocomposite }\end{array}$ & $\begin{array}{l}\text { Aufnahme } \\
\text { von } 4 \mathrm{Nr} \text {-2- } \\
\text { Fäden }\end{array}$ & knotenfrei & $3,9 \mathrm{~mm} / 4,5 \mathrm{~mm}$ & $\begin{array}{l}4,75 \mathrm{~mm} / \\
5,5 \mathrm{~mm}\end{array}$ & $19,1 \mathrm{~mm}$ & $\begin{array}{l}\text { laterale Nahtreihe, } \\
\text { einzuschrauben }\end{array}$ \\
\hline
\end{tabular}

PEEK - Polyetheretherketon; PLLA - Poly-L-Laktid-Säure; HA - Hydroxylapatit; PLDLA - Poly-L/D-Laktid-Säure; TCP - Tri Calcium Phosphat; BR - BIOCRYL ${ }^{\circledR}$ RAPIDETM Material (TCP/ PLGA bestehend aus Poly-L-Laktid-Co-Glycol-Säure; Biocomposite - Tri Calcium Phosphat und Poly-L/D-Laktid-Säure 
$0,981 \mathrm{~kg} \approx 1 \mathrm{~kg}$ ). Anschließend erfolgte die zyklische Belastung zwischen 10 und $100 \mathrm{~N}$ mit einer Frequenz von 0,5 Hz. Dieses Testprotokoll lief für 200 Zyklen oder bis zum Materialversagen. Nach 200 Zyklen erfolgte die Prüfung der maximalen Ausreißfestigkeit mit einer Zuggeschwindigkeit von $12,5 \mathrm{~mm}$ pro Sekunde. Sämtliche Testdaten wurden pro Sekunde protokolliert inkl. der notwendigen Anzahl von Belastungszyklen bis zu einer Dislokation der Anker von 5 und $10 \mathrm{~mm}$.

Als Testendpunkte wurde die Dislokation nach 100 und 200 Zyklen festgehalten, falls möglich die maximal aufgebrachte Zugkraft bis zum Implantatversagen sowie die Ursache des Implantatversagens (knöchernes Ausreißen des Ankers, Ausreißen der Öse im Anker oder Reißen des Fadens). Bemerkenswert ist, dass die getesteten Ankersysteme vergleichbare Ausreißfestigkeiten boten (Erläuterung im nachfolgenden Abschnitt).

Für die meisten Ankersysteme sind unterschiedliche Dimensionen bzw. Durchmesser verfügbar, wobei Tab. 1 einen Überblick in Bezug auf Material, Dimension und spezifischer Einsatzmöglichkeit (mediale oder laterale Nahtreihe) ermöglicht. An Ankermaterialien werden bspw. Titan [Ti] (Titan mit Zusätzen von Aluminium, Vanadium, Molybdän, etc.), Hydoxylapatit [HA], Poly-L-Laktit [PLLA], Trikalziumphosphat [ $\beta$-TCP], Polylaktid Co-Glycolid [PLGA], PEEK (Polyether ether ketone) Biocomposite bzw. Kombinationen aus resorbierbarem Material angeboten.

Fadenanker und knotenlose Anker können ein- oder 2-reihig implantiert werden

Nachfolgend werden besondere Aspekte der einzelnen Ankersysteme erläutert.

Corkscrew-FT-Fadenanker (Arthrex, Naples, FL, USA): Dieses Ankersystem steht mit 3 unterschiedlichen Dimensionen zur Verfügung (4,5, 5,5 und 6,5 mm). Zusätzlich können diese aus Titan, PEEK, PLLA und Biocomposite (85\% PLLA und $15 \% \beta$-TCP) geliefert werden. Jeweils 2 Fäden liegen in einer Öse, um ein besonders leichtes Gleiten des Knotens zu ermöglichen. Die Implantation erfolgte nach Eröffnen der Kortikalis mit einem Speer bzw. Dorn, um anschließend das Gewinde zu schneiden und den Anker einzudrehen. Das Gewinde ist insbeson- dere für schlechte Knochenqualität entwickelt worden und ist gut für den spongiösen Knochen geeignet. Durch die unterschiedlichen Durchmesser stehen bei schlechtem Halt Rückzugsmöglichkeiten auf größere Durchmesser zur Verfügung.

Corkscrew-Fadenanker (Arthrex): Diese Anker sind sowohl in Titan als auch aus PLDLA verfügbar. Diese Anker zeichnen sich durch ein besonderes Spongiosagewinde mit einem sehr dünnen Kerndurchmesser und großer Gewindesteigung aus. Die Fadenöse liegt an der Ankerbasis und besteht aus einem Faden in der PLDLA-Version für ein besonders reibungsarmes Fadengleiten.

PushLock und PushLock SP (Arthrex): Dieser knotenlose Anker ist sowohl für RM-Rekonstruktion als auch für die glenohumerale Stabilisierung geeignet und steht in 2 Dimensionen zur Verfügung (3,5 und 4,5 mm). Diese Anker werden eingeschlagen (tap-in). Drei unterschiedliche Materialien stehen zur Verfügung mit PLLA, PEEK und Biocomposite. Die SP-Version dieses Ankers beinhaltet eine Titanöse an der Ankerspitze, um auf die Präparation mit einem Speer zu verzichten und Zeit einzusparen.

SwiveLock C und SwiveLock SP (Arthrex): Dieses Ankersystem hat ein dem PushLock-System ähnliches Verankerungsprinzip und wird eingeschraubt (screw-in). Es stehen 2 Dimensionen mit 4,75 und 5,5 $\mathrm{mm}$ im Durchmesser zur Verfügung. Diese Anker sind in PEEK, PLLA und Biocomposite verfügbar. Auch die SP-Variante dieses Ankersystems besteht aus einer Titanöse an der Ankerspitze, um einen Präparationsschritt einzusparen.

SwiveLock (Arthrex): Diese Anker sind in 4,75 und 5,5 mm verfügbar und aus PEEK und PLLA erhältlich. Der Unterschied zu den Versionen $C$ und SP liegen in einer offenen bzw. geschlitzten Fadenöse. Diese Variante ermöglicht für besondere Konstellationen mehr Flexibilität für die Fertigstellung der 2. Nahtreihe.

Reel X (Stryker, Duisburg): Dieser knotenlose Anker hat einen äußeren Durchmesser von $5,5 \mathrm{~mm}$, der sich im Rahmen der Implantation auf 6,5 mm expandieren lässt. Der Anker besteht aus PEEK und einem inneren Blockierungsmechanismus. Eine selbst Speer ermöglicht auch die Implantation ohne Benutzung der zusätzlichen Implantationsinstrumente. Zwei Nr-2-(USP-)Fäden liegen ein. Nach Implantation des Ankers wird durch Drehen am Ankerhandgriff (1-3 volle Umdrehungen) die Blockierung des im Anker einliegenden Stahlstifts realisiert. Durch die Rotation dieses Stiftes im Ankerinneren werden die Fäden bis zu $9 \mathrm{~mm}$ näher an die Ankerspitze geschoben mit gleichzeitiger Expansion des Ankers und dadurch bedingter Zunahme der Ausreißkraft. Hier befindet sich somit auch die Fadenöse.

Footprint Ultra PK (Smith \& Nephew Endoscopy, Andover, MA, USA): Dieser Anker ist in 4,5 und 5,5 mm erhältlich und besteht aus PEEK. Er beinhaltet einen geflochtenen 2-0-(USP-)Polyesterfaden. Der 5,5-mm-Anker benötigt einen 4,5$\mathrm{mm}$ - und der 4,5-mm-Anker einen 3,5mm-Gewindeschneider. Alternativ können beide Anker mit Nutzung einer 3,8mm-Ahle implantiert werden. Die Fadenöse befindet sich zentral nahe der Ankerspitze.

TwinFix-Anker (Smith \& Nephew Endoscopy): Diese Anker sind in 3 Dimensionen erhältlich $(4,5,5,5$ und $6,5 \mathrm{~mm})$ sowie in 3 unterschiedlichen Materialien (PEEK [PK], Hydroxylapatit [HA] und Ti$\tan [\mathrm{Ti}])$. In Abhängigkeit von der Ankerdimension und dem Material werden 2 oder 3 Fäden eingesetzt. Diese haben die Stärke 2 (USP) in weißer, blauer und schwarzer Farbgebung. Der HA-Anker besteht aus einer Poly-L-Lactid- und Hydroxylapatit-Mischung und sollte nicht im harten Knochen implantiert werden (Brechen des Ankers möglich). Die TwinFix-Anker benötigen je einen $1 \mathrm{~mm}$ im Durchmesser dünneren Gewindeschneider im Rahmen der Implantation. Die 5,5-mm- und 6,5-mm-Anker können auch mit einem 5,5-mm/6,5-mm-Speerdilatator und der 4,5-mm-Anker mit einem 4,5-mm-Speerdilatator implantiert werden. Eine Querverstrebung des ansonsten kanülierten Ankers bildet nahe an der Ankerspitze die Fadenöse.

Healicoil-Anker (Smith \& Nephew Endoscopy): Diese Anker haben ein besonderes Merkmal durch vorhandene Perforationen des Ankerkerns (offene Ankerarchitektur), um dem umgebenden Knochen ein Einwachsen zu ermöglichen, und reduzieren das verwendete Material, welches im Knochen zurückbleibt. Die Anker sind in 4,5 und 5,5 mm verfügbar und bestehen aus PEEK. Eine Querverstrebung des kanülierten Ankers bildet an der Ankerspitze die Fadenöse. Der 5,5-mm-Anker ist auch mit 3 Fäden verfügbar. 
Morphix (Medshape Solutions): Dieser Anker ist mit 4,5 und 5,5 mm für die RMRekonstruktion verfügbar. Zwei Fäden der Stärke 2 (USP) beinhalten die Anker. Dieses Ankersystem zeichnet sich durch einen selbstspreizenden Mechanismus aus, sodass sich der einzuschlagende Anker subkortikal stabil verklemmt. Mit einem Bohrer wird der Insertionsort vorbereitet und der Anker implantiert. Durch eine Viertelumdrehung gegen den Uhrzeigersinn wird der Anker im Setzinstrument freigegeben, um ihn dann mit einem Schlegel subkortical aus dem Setzinstrument gänzlich zu lösen.

CrossFT BC (ConMed-Linvatec, Largo, FL, USA): Diese Anker sind in 3 Dimensionen verfügbar $(4,5,5,5$ und $6,5 \mathrm{~mm})$. Sie bestehen aus einem Biocomposite-Material sowie 2 Fäden. Die Gewindesteigung nimmt nicht von der Ankerspitze zur Basis ab, um sowohl im spongiösen als auch im kortikalen Knochen größtmögliche Ausreißfestigkeit zu erzielen. Dimensionsgerechte Speere bzw. Implantationsinstrumente stehen zur Verfügung.

JuggerKnot $2.8 \mathrm{~mm}$ (Biomet Sport Medicine, Warsaw, IN, USA): Dieses Ankersystem basiert auf einem 2,8 mm messenden Faden ohne klassische solide Ankeranteile. Der Anker besteht aus 2 Fäden der Stärke 2 für die Rekonstruktion und einer $2 \mathrm{~mm}$ weiten und $25 \mathrm{~mm}$ langen Stulpe. Letztere wird subkortikal so zusammengeknäult, dass dieser Fadenknödel subkortikal verankert ist. Dieser Anker wird nach Anlegen eines Bohrlochs $(2,9 \mathrm{~mm})$ in den Knochen eingeschlagen.

Quattro X Quattro Link (Cayenne Medical, Arizona, USA): Diese beiden Anker bestehen aus PEEK und können in 4,5 und 5,5 mm genutzt werden. Der Quattro $\mathrm{X}$ ist für die 1 -reihige oder mediale Reihe der 2-reihigen Naht gedacht und verfügt über 2 Fäden. Die Öse des kanülierten Ankers wird durch eine Querverstrebung nahe der Ankerspitze gebildet. Der Quattro-Link-Anker ist ein knotenloser Anker mit einer Öse, die bis zu 8 Fäden aufnehmen kann und dadurch besonders für die 2 . Nahtreihe geeignet ist.

Healix (DePuySynthes, Leeds, GB): Diese Anker sind in 3 Dimensionen verfügbar $(4,5,5,5$ und $6,5 \mathrm{~mm})$. Diese stehen als PEEK, Titan oder Biocryl Rapid (30\% $\beta$-TCP und $70 \%$ aus PLGA) zur Verfügung. Jeweils 2 oder 3 Fäden liegen ein. Zur Implantation wird ein an die Dimension angepasster Speer-Gewindeschneider genutzt. Die Gewindesteigung nimmt von der Spitze zur Basis ab, sodass dieser Anker sowohl im kortikalen als auch spongiösen Knochen stabil verankert werden kann. Eine Querverstrebung des kanülierten Ankers bildet an der Ankerspitze die Fadenöse.

\section{Stabilitätstest}

Zyklische Tests erfolgten durch Barber et al. 2013. In den Jahren zuvor (20062011) erfolgte die Bestimmung der maximalen Ausreißkraft [4]. Die aktuellsten Stabilitätstests zeigten, dass die Ausreißfestigkeit insgesamt hoch ist [1]. Nach 100 und 200 Zyklen zeigte sich für alle Anker keine Dislokation von mehr als $3 \mathrm{~mm}$. Ein Herausziehen (Pull-out) der Anker kam bei Überprüfung der Maximalhaltekraft nur selten vor (ca. $12 \%$ ). Die Ankerösen versagten in ca. 55\% der Fälle. Das Fadenmaterial (UHMWPE) versagte in ca. 33\% der Fälle. Fasst man diese und weitere biomechanische Untersuchungen zusammen, zeigen sich ähnliche Stabilitätsmerkmale der unterschiedlichen Ankersysteme. Interessanterweise zeigte sich für alle Anker statistisch kein relevanter Unterschied in Bezug auf die kortikale und spongiöse Ausreißfestigkeit. Erfahrungen bzw. Vergleiche mit osteoporotischem Knochen liegen jedoch bei diesem Testprotokoll nicht vor [1].

Bei dieser großen Auswahl stellt sich dem Chirurgen die Frage, in welcher Situation welcher Anker bzw. welches Ankerdesign besonders geeignet erscheint und ob Anker zwangsläufig notwendig sind oder nicht, bzw. ob es auch Situationen gibt, die ohne Fadenanker beherrscht werden können?

Einige biomechanische Untersuchungen zeigen, dass insbesondere die 2-reihige (double-row) RM-Rekonstruktion der klassischen transossären Naht hinsichtlich der Stabilität, Steifigkeit und Haltekraft überlegen ist $[2,6]$. Zudem kann durch die Verwendung von Fadenankern der operative Zugang verkleinert werden (mini-open Technik) oder gänzlich arthroskopisch erfolgen. Eine transossäre RM-Rekonstruktion ist unter Verwendung spezieller Instrumente allerdings auch möglich, wobei die klinischen Ergebnisse und Erfahrungen bisher begrenzt sind. Fadenanker und knotenlose Anker können beide sowohl für die mediale als auch laterale Nahtreihe, aber auch jeweils singulär in der 1-reihigen Technik oder kombiniert in der 2-reihigen Technik eingesetzt werden. Bio- mechanische Untersuchungen der 2-reihigen Technik zeigten im Kombinationsvergleich Fadenanker/knotenloser Anker (FA/KA) und knotenloser Anker/knotenloser Anker (KA/KA), dass die FA/KAKombination steifer ist und das Lastverhältnis medial zu lateral 2:1 beträgt [7]. Diese Lastverteilung könnte in einigen Fällen zum klinischen und ggf. auch biomechanischem Versagen führen, obwohl die Gesamtstabilität (Steifigkeit, Spaltbildung und maximale Haltekraft) dieser Ankerkombination (medial FA/lateral KA) bisher am besten ausfiel $[8,9]$. Um die Sehnenretraktion im nachfolgenden Text einheitlich bzw. standardisiert wiederzugeben, wird diese in 3 Stufen eingeteilt: spannungsfrei/keine Muskelatrophie (-), leichte Spannung/diskrete Muskelatrophie $(+)$ und mäßige Spannung/ mäßige Muskelatrophie (++).

Nachfolgend werden konkrete klinische Konstellationen beschrieben und die mögliche Rekonstruktionstechnik mit und ohne Anker dargestellt. Dabei gehen wir zunächst auf begrenzte RM-Läsionen (Bateman 1 und 2) ein, um im 2. Block größere Läsionen (Bateman 3 und 4) zu diskutieren:

Die 2-reihige Rekonstruktion ist biomechanisch steifer und hat höhere Ausreißkräfte.

Bateman 1 und 2: Die Indikation zur klassischen transossären Naht sollte auf unproblematische RM-Läsionen begrenzt werden, wie etwa Bateman-1-2Läsionen ohne größere Sehnenretraktion (Patte 1). Falls diese stärker ausgeprägt vorliegt (Patte 2), muss intraoperativ der Muskelhub bzw. die Muskelverkürzung geprüft werden. Bei nicht atrophierter Muskulatur und spannungsarmer Rekonstruktion (-) kann die transossäre Naht erfolgen. Für solche Situationen sind natürlich auch alle Nahtanker geeignet. Konkret kann für diese Fälle sowohl die 1-reihige Naht mit FA oder KA als auch die 2-reihige Naht mit der Kombination FA/KA oder KA/KA erfolgen. Falls die Sehnenrekonstruktion bzw. Reposition an den Footprint nur mit leichtem Zug möglich ist $(+)$, sollte keine transossäre Naht erfolgen, sondern die 2-reihige Naht mit der Kombination FA/KA oder KA/KA erfolgen. Im Falle einer mäßigen Spannung $(++)$ sollte die 2-reihige Rekonstruktion mit der Kombination FA/KA erfolgen.

Bateman 3 und 4: Für solche großen RMLäsionen sollte keine transossäre Naht 


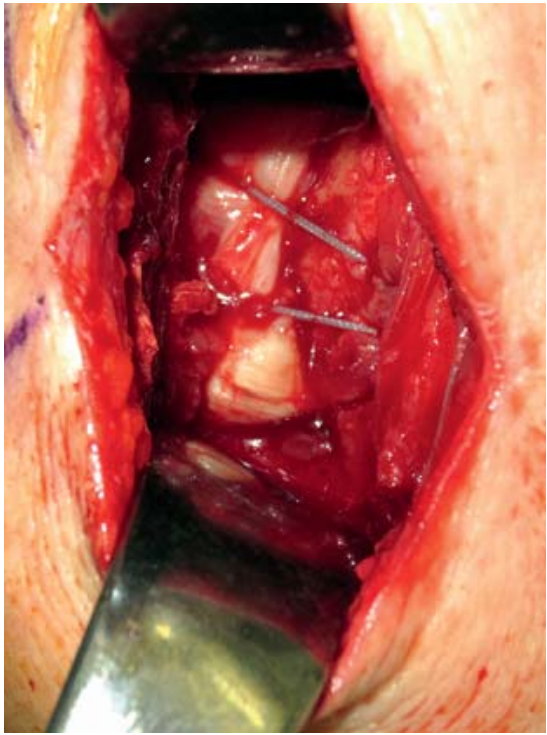

Abb. 3 Beispiel einer Rekonstruktion bei Bateman-4-Läsion in 2-reihiger Technik. Miniopen-Rekonstruktion einer Bateman-4-Läsion in 2-reihiger Technik, wobei medial 3 Fadenanker und lateral 3 knotenlose Anker genutzt wurden, um die mäßige Spannung auf eine größere Fläche zu verteilen (Inzision $5 \mathrm{~cm}$ messend).

erfolgen, da durch die Verwendung der Nahtanker nicht nur der Zugang kleiner wird, sondern auch eine relevante Zeitersparnis möglich ist bei biomechanischen Vorteilen. Wenn die Rekonstruktion spannungsarm (-) möglich ist, kann auch die 1-reihige Rekonstruktion bei guter Sehnenqualität erwogen werden, wobei die Last auf 3 Anker (FA oder $\mathrm{KA})$ verteilt werden sollte. Im eigenen Vorgehen bevorzugen wir jedoch die 2reihige Naht mit der Kombination FA/KA oder KA/KA. Bei leichter Sehnenspannung $(+)$ sollte in Abhängigkeit von der Sehnenqualität die 2-reihige Naht erfolgen mit den Ankerkombinationen FA/KA oder KA/KA. Bei mäßiger Spannung sollte auf die 2-reihige Naht mit FA/KA zurückgegriffen werden, aufgrund der biomechanisch stabilsten Situation (Abb. 3, Beispiel einer Rekonstruktion bei Bateman-4-Läsion in 2-reihiger Technik).

Bei diesem Gesamtkontext darf die Knochenqualität des Tuberculum majus und minus nicht vergessen werden. Bei adäquat harter Qualität der Kortikalis und Spongiosa des Oberarmkopfs kann für die erwähnten Konstellationen die transossäre Naht erfolgen. Bei osteoporotischer Knochenqualität sollte grundsätzlich keine transossäre Naht erfolgen, da die Sehnenrekonstruktion mit Ankern überlegen ist. Nicht selten kommt es zu der Konstellation mit Zysten im Oberarmkopf, die man technisch bei rekonstruktionswürdigem Schultergelenk (Sehnenqualität/keine oder geringe Muskelatrophie) mit einer Spongiosaplastik und 2-reihigen Naht (FA/KA) beherrschen kann [10]. Man muss den Knochendefekt mit einer ausreichenden Menge von spongiösen Knochen so verdichten, dass die einzubringenden Anker stabil einliegen. Hierzu sollte man großzügig auf allogene Spongiosa zurückgreifen, da die Qualität und Quantität vom patienteneigenen Beckenkamm unter Berücksichtigung des Patientenalters häufig reduziert ist.

\section{Schlussfolgerungen}

Zur Rekonstruktion von RM-Läsionen haben sich Nahtanker gegenüber der klassischen transossären Naht durch stabilere Rekonstruktionen, Zeitersparnis und kleinere Zugänge bis hin zur arthroskopischen Technik bewährt. Einheitliche biomechanische Untersuchungen bzw. Testprotokolle liegen nicht für alle verfügbaren Anker vor. Die vorliegenden Ergebnisse zeigen, dass die Anker vergleichbare Stabilitätsverhältnisse ermöglichen, sodass eine generelle Empfehlung nicht ohne Weiteres ausgesprochen werden kann. Diesbez. muss auch erwähnt werden, dass das schwächste Glied der Rekonstruktion weniger die Anker und das Nahtmaterial sind, sondern das Faden-Sehnen-Interface mit der Möglichkeit des Ausreißens des Nahtmaterials aus dem Sehnengewebe, insbesondere bei eingeschränkter RM-Sehnenqualität. Daher muss nicht zwangsläufig primär auf besonders kräftige Anker zurückgegriffen werden, da der Stabilitätsgewinn keinen effektiven Vorteil bietet und zusätzlich die Revision in solchen Fällen aufgrund größerer Knochendefekte erschwert ist. Grundsätzlich sollte die Rekonstruktionstechnik und die damit verbundene Ankerwahl bzw. Kombination intraoperativ individuell ausgewählt werden, sodass sich in der Praxis eine operationstechnische Flexibilität bewährt hat.

\section{Literatur}

1 Barber FA, Herbert MA. Cyclic loading biomechanical analysis of the pullout strengths of rotator cuff and glenoid anchors: 2013 update. Arthroscopy 2013; 29: 832-844

2 Cole BJ, ElAttrache NS, Anbari A. Arthroscopic rotator cuff repairs: an anatomic and biomechanical rationale for different suture-anchor repair configurations. Arthroscopy 2007; 23: 662-669

${ }^{3}$ Barber FA, Hapa O, Bynum JA. Comparative testing by cyclic loading of rotator cuff suture anchors containing multiple high-strength sutures. Arthroscopy 2010; 26 (Suppl.9): S134-S141

${ }^{4}$ Barber FA, Herbert MA, Hapa 0 et al. Biomechanical analysis of pullout strengths of rotator cuff and glenoid anchors: 2011 update. Arthroscopy 2011; 27: 895-905

5 Gerber C, Schneeberger AG, Beck $M$ et al. Mechanical strength of repairs of the rotator cuff. J Bone Joint Surg Br 1994; 76: 371-380

6 Salata MJ, Sherman SL, Lin EC et al. Biomechanical evaluation of transosseous rotator cuff repair: do anchors really matter? Am J Sports Med 2013; 41: 283-290

7 Vaishnav S, Millett PJ. Arthroscopic rotator cuff repair: scientific rationale, surgical technique, and early clinical and functional results of a knotless self-reinforcing doublerow rotator cuff repair system. J Shoulder Elbow Surg 2010; 19 (Suppl. 2): S83-S90

8 Mazzocca AD, Millett PJ, Guanche CA et al. Arthroscopic single-row versus double-row suture anchor rotator cuff repair. Am J Sports Med 2005; 33: 1861-1868

9 Yamakado K, Katsuo S, Mizuno Ket al. Medialrow failure after arthroscopic double-row rotator cuff repair. Arthroscopy 2010; 26: 430 435

10 Burkhart SS, Klein JR. Arthroscopic repair of rotator cuff tears associated with large bone cysts of the proximal humerus: compaction bone grafting technique. Arthroscopy 2005; 21: 1149

\section{Dr. med. Atesch Ateschrang}

Oberarzt und Leitender Arzt der Sektion Sporttraumatologie und arthroskopischen Chirurgie

\section{Dr. med. Stephan Reidmann}

Assistenzarzt

Dr. med. Steffen Schröter

Facharzt für Orthopädie und Unfallchirurgie

Prof. Dr. med. Ulrich Stöckle

Ärztlicher Direktor

Berufsgenossenschaftliche

Unfallklinik Tübingen

Klinik für Unfall- und

Wiederherstellungschirurgie

Eberhard Karls Universität Tübingen

Schnarrenbergstraße 95

72076 Tübingen

ate21@gmx.de 